\title{
Interventions visant l'application des connaissances en pratique pharmaceutique
}

\author{
par Apolline Adé, Denis Lebel et Jean-François Bussières
}

\section{RÉSUMÉ}

Contexte : La littérature scientifique portant sur l'application des connaissances (AC) est vaste et complexe et les publications sur les interventions dans le domaine de la santé concernent surtout les médecins et les infirmières. Pour autant que les auteurs sachent, il n'existe pas de revue documentaire s'intéressant à l'AC et à ses retombées en pharmacie.

Objectif : Décrire le profil des interventions visant l'AC en pratique pharmaceutique.

Source des données : La plateforme Knowledge Translation+ $(\mathrm{KT}+)$ a été utilisée pour en extraire des articles publiés entre janvier 2010 et décembre 2016 à l'aide du terme "pharmacist ".

Sélection des études et extraction des données : Les principales variables retenues pour établir le profil des interventions visant l'AC en pratique pharmaceutique étaient le protocole de recherche de l'étude, le lieu de l'intervention, les rôles du pharmacien, les types de connaissances transférées et les retombées. Le codage de la nature des interventions pharmaceutiques reposait sur la classification du site Impact Pharmacie.

Synthèse des données : Au total, 114 articles ont été sélectionnés : revues systématiques ( $n=25,22 \%)$, études contrôlées à répartition aléatoire ( $n=45,40 \%)$ études rétrospectives $(n=21,18 \%)$, études prospectives ( $n=13,11 \%)$, études pré-post intervention $(n=10,9 \%)$. Les études se déroulaient surtout en établissement de santé (74\%). La majorité des interventions ciblaient des étapes de soins pharmaceutiques et la réalisation de séances d'éducation thérapeutique et de conseils prodigués aux patients. Il existait un manque de rigueur méthodologique lors de la conception des interventions et quant à leur description.

Conclusion : Le pharmacien est le principal producteur de connaissances et oriente les interventions visant leur application vers les patients ou les professionnels de santé. Celles-ci concernaient principalement la démarche de soins pharmaceutiques et le travail en interdisciplinarité. La mise en place d'une formation initiale et continue, la gestion de l'information et la désignation d'un pharmacien responsable de l'AC au sein de chaque département de pharmacie pourraient encourager le développement de cette mise en application des connaissances. Ce concept peut être utile pour soutenir la création d'un modèle de pratique pharmaceutique cohérent.

Mots clés : intervention, pharmacien, pharmacie, application des connaissances, transfert des connaissances

\begin{abstract}
Background: The scientific literature on knowledge translation (KT) is vast and complex, and most publications concerning health care interventions involve physicians and nurses. To the authors' knowledge, there have been no literature reviews on KT and its impact on pharmacy practice.
\end{abstract}

Objective: To determine the profile of interventions relating to KT in pharmacy practice.

Data Sources: The term "pharmacist" was used to search the web platform Knowledge Translation+ $(\mathrm{KT}+)$ to identify pertinent articles published between January 2010 and December 2016.

Study Selection and Data Extraction: The main variables analyzed to determine the profile of KT interventions in pharmacy practice were the study's research protocol, the geographic location of the intervention, pharmacist roles, the types of knowledge transferred, and impacts of the interventions. The nature of pharmacy interventions was coded according to the classification on the Impact Pharmacie website.

Data Synthesis: A total of 114 articles were selected: systematic reviews $(n=25,22 \%)$, randomized controlled trials $(n=45,40 \%)$, retrospective studies $(n=21,18 \%)$, prospective studies $(n=13,11 \%)$, and pre-post intervention studies $(n=10,9 \%)$. Most of the studies $(74 \%)$ were conducted in a health care institution. The majority of interventions targeted pharmaceutical care steps, therapeutic educational sessions, and patient education. There was a lack of methodological rigour during the development of interventions and in their description.

Conclusion: Pharmacists are key generators of knowledge, and their interventions related to KT are directed toward patients or other health care professionals. These interventions have mainly addressed the pharmaceutical care process and interdisciplinary work. The implementation of initial and continuing education, the management of information, and the designation of a pharmacist responsible for KT in each pharmacy department might promote the development of such KT. This concept might in turn support the design of a coherent pharmacy practice model.

Keywords: intervention, pharmacist, pharmacy, knowledge translation, knowledge transfer

J. Can. Pharm. Hosp. 2018;71(3):187-95 


\section{INTRODUCTION}

T 'application des connaissances (AC) est un processus visant Là favoriser l'utilisation des données de la recherche dans la pratique des professionnels de la santé pour améliorer la qualité et la sécurité des soins offerts aux patients. Il s'agit d'un processus dynamique, bidirectionnel et itératif, d'échange et de transfert de preuves, d'information et de données entre les producteurs et les utilisateurs des connaissances ${ }^{1}$. Les interventions représentent un élément-clé du processus d'AC, car elles permettent la mise en application concrète des connaissances ${ }^{2}$. Dans la littérature scientifique, le terme "intervention » est utilisé de façon interchangeable avec le terme "stratégie de mise en œuvre ". D'après Curran et collab., la stratégie de mise en œuvre constitue une méthode efficace pour améliorer l'adoption, la mise en œuvre et la durabilité d'un programme clinique ou de pratiques cliniques ${ }^{3}$. Il existe différents types d'interventions (p. ex. simples c. multiples, actives c. passives) mais il n'existe pas de classification unique de celles-ci. En effet, la plateforme WhatisKT recense vingt-trois taxonomies et huit listes classifiant les interventions ${ }^{4}$. Une des classifications les plus utilisées est celle du groupe EPOC, coordonné depuis 1997 par l'équipe de Grimshaw Shepherd et collab. qui recense des études évaluant l'efficacité d'interventions professionnelles destinées à promouvoir les changements de pratique et l'AC 5 .

Jusqu’à maintenant, les chercheurs n’ont pas réussi à déterminer la stratégie ou la combinaison de stratégies la plus efficace. On admet que chaque contexte requiert une stratégie sur mesure. Ainsi, il faut notamment tenir compte des organisations, des acteurs impliqués et des caractéristiques des connaissances à transférer avant de développer une stratégie pour maximiser les chances de succès ${ }^{6}$. La revue systématique de Grimshaw et collab. publiée en 2004 montre que les réunions éducatives et les interventions de rappels semblent être les interventions les plus efficaces alors que l'appui de leaders d'opinion, la distribution de matériel éducationnel et les audits avec rétroaction semblent avoir une efficacité plus limitée ${ }^{7}$. Les interventions multiples ne sont pas forcément plus efficaces que les interventions simples ${ }^{7,8}$. Dans une revue systématique, LaRocca et collab. ont démontré que les stratégies multiples avaient entrainé des changements du niveau des connaissances des participants mais pas des pratiques? ${ }^{9}$. Ils ont également montré que les stratégies passives (p. ex. utilisation de supports imprimés) sont souvent moins efficaces. À partir d'une analyse de 33 revues de littérature publiées, Prior et collab. ont conclu que les interventions les plus efficaces étaient diversifiées et comportaient notamment des sessions éducatives et des rappels ${ }^{10}$.

Le pharmacien est plus que jamais au cœur du circuit du médicament, comme soignant de première ligne tant en officine qu'en pharmacie hospitalière, et il possède une vue d'ensemble de toute la pharmacothérapie du patient tout en détenant une solide expertise en matière de médicament. Ainsi le processus d'AC présente de nombreux intérêts pour le pharmacien, dont le rôle n'est plus seulement de dispenser des médicaments mais aussi de gérer les échanges de connaissances au sens général du terme entre les patients et les professionnels de santé et de favoriser leur mise en pratique.

La littérature scientifique portant sur l'AC est vaste et complexe et les publications sur les interventions dans le domaine de la santé concernent surtout les médecins et les infirmières ${ }^{11}$. À notre connaissance, il n'existe pas de revue documentaire s'intéressant à l'AC et à ses retombées en pharmacie.

\section{MÉTHODES}

Il s'agit d'une revue de la littérature dont l'objectif principal est de décrire le profil des interventions pharmaceutiques ayant pour objet l'AC. Entreprendre une recherche bibliographique sur l'AC n'est pas simple. D'abord, la terminologie associée au processus est très diverse et variable (différents termes peuvent désigner le même concept) et il existe de nombreuses définitions pour chaque terme. Ensuite, la littérature sur l'AC est complexe, car il s'agit d'une discipline nouvelle liée à de nombreuses autres disciplines (p. ex. sociologie, anthropologie, philosophie), le volume d'articles publiés est très large, la recherche d'articles est chronophage et enfin, il n'est pas évident de déterminer quelle est la meilleure ressource à utiliser.

Afin de décider des articles portant sur les interventions pharmaceutiques en $\mathrm{AC}$, nous avons utilisé la plateforme Knowledge Translation+ (KT+). KT + est une banque de données du Health Information Research Unit de McMaster University au Canada ${ }^{12}$. Ce projet d'AC est soutenu par les Instituts canadiens de recherche en santé (IRSC). Cette plateforme recense les articles et documents relatifs au domaine de l'AC et à celui de l'amélioration de la qualité des soins. Une équipe de recherche évalue des articles originaux et des revues de systématiques sur le sujet provenant de 122 journaux y compris la bibliothèque Cochrane. Toutefois, aucun des journaux retenus ne porte spécifiquement sur les pratiques pharmaceutiques ${ }^{13,14}$. La banque de données permet deux types de consultation, soit des articles satisfaisant aux critères d'inclusion du projet, quality-filtered $K T$ articles, et des articles sélectionnés et recensés par l'équipe mais qui n’ont pas été évalués non-quality filtered KT articles. Les articles sélectionnés dans ces deux catégories doivent présenter un contenu pertinent par rapport aux interventions d'AC. Toutefois, seuls les quality-filtered $K T$ articles sont classés selon la pertinence clinique de leur contenu et la rigueur de la méthodologie. Le classement est réalisé par trois membres d'un panel international de professionnels de santé s'intéressant à l'AC. La base de données est continuellement mise à jour.

Une stratégie de recherche à partir du terme "pharmacist» a été menée dans la plateforme KT + sur une période de sept ans, soit de janvier 2010 à décembre 2016. Le terme "pharmacist" a été préféré au terme "pharmacy ", car ce dernier n'est pas assez précis pour rechercher des articles ayant pour thème principal le rôle du pharmacien. Le terme "pharmacist " présentait l'avantage de cibler des articles portant sur les interventions du pharmacien. 
De plus, nous avons choisi d'effectuer la recherche sur une période postérieure à la publication de la définition de l'AC par les IRSC (2008) afin de mesurer la fréquence de la mention de ce processus dans les études.

La recherche dans cette plateforme a été privilégiée à une recherche plus traditionnelle dans les banques de données Pubmed ou Google Scholar, car les recherches d'études originales sur des interventions d'AC sont difficiles à découvrir, faute de termes ciblés dans le vocabulaire et compte tenu de leur codification non spécifique à la pharmacie.

À partir des articles retenus, nous avons établi le profil des interventions pharmaceutiques ayant pour objet l'AC en extrayant les variables suivantes : pays, protocole de recherche, lieu de l'intervention, les interventions (c.-à-d. producteurs / courtiers de connaissances, utilisateurs de connaissances), les pathologies concernées par les interventions, les interventions elles-mêmes (c.-à-d. catégories d'interventions, rôles du pharmacien, types de connaissances transférées), les répercussions (c.-à-d. objectifs primaires évalués, impact des interventions, soit positif, neutre, négatif). Les rôles du pharmacien et les pathologies ont été codifiés après une analyse des données recueillies. La nature des interventions pharmaceutiques a été codifiée selon la classification proposée sur le site Impact Pharmacie ${ }^{15}$. En effet, la classification EPOC, habituellement utilisée pour décrire les interventions professionnelles dans le domaine de la santé, n'est pas adaptée pour décrire les interventions pharmaceutiques. Le site Impact Pharmacie recense les preuves relatives aux rôles et aux répercussions de l'activité du pharmacien et classe les interventions pharmaceutiques en neuf catégories.

À partir des données recueillies, nous avons établi un profil des interventions pharmaceutiques et déterminé des pistes de réflexion entourant l'AC en pharmacie.

Seules des statistiques descriptives ont été réalisées.

\section{RÉSULTATS}

$\mathrm{Au}$ total, 114 articles portant sur des interventions pharmaceutiques ayant pour objet l'AC ont été retenus (pour la liste complète des articles, voir l'annexe 1 , publiée au https://www.cjhp-online.ca/index.php/cjhp/issue/view/155/ showToc). Ils représentent $17 \%$ (114/666) des articles de la plateforme KT+ recensés lorsque la recherche s'effectue avec le terme "pharmacist». La figure 1 représente un diagramme de flux des articles sélectionnés pour l'analyse. Le critère d'inclusion principal était le thème des articles, c'est-à-dire qu'ils devaient évoquer des interventions pharmaceutiques visant l'AC.

Des 114 articles retenus, on trouve 25 revues systématiques (22\%), 45 études contrôlées à répartition aléatoire (39\%), 21 études rétrospectives (18\%), 13 études prospectives (11\%), 10 études pré- post intervention (9\%). Plus des deux-tiers $(n=79$, $69 \%$ ) des études originales recensées sont incluses dans au moins une revue systématique.

Parmi les articles inclus, 29 sont qualifiés de quality-filtered $K T$ articles, dont 21 revues systématiques et huit articles originaux, et 85 articles sont qualifiés de non-quality filtered $K T$ articles, dont quatre revues systématiques et 81 articles originaux. Nous n'avons pas tenu compte de la distinction entre qualityfiltered $K T$ articles et non-quality filtered KT articles au cours de notre analyse, car tous les articles répondaient au critère principal d'inclusion.

\section{Études originales}

Des 114 études retenues, 89 sont des études originales. Soixante-treize pour cent $(n=65)$ des études proviennent des États-Unis, $7 \%(n=6)$ du Canada, $6 \%(n=5)$ du Royaume Uni, $3 \%(n=3)$ des Pays-Bas et $11 \%(n=10)$ d'autres pays. Lexpression "knowledge translation", traduite par " application des connaissances ", n’a toutefois été trouvée que dans une seule étude ${ }^{16}$.

Le tableau 1 présente un profil des interventions pharmaceutiques mettant en valeur l'AC.

Les études se déroulent surtout en établissement de santé (74\%), y compris les centres hospitaliers universitaires, les centres de soins de longue durée, les cliniques privées, les établissements pour anciens combattants.

Dans ces études, le pharmacien est producteur ou courtier des connaissances dans $94 \%$ des études tandis que ce rôle est confié à d'autres professionnels de santé dans $6 \%$ des cas. Le producteur de connaissances crée les connaissances (p. ex. en tant que chercheur) et les synthétise sous forme de message clair adapté au public visé. Par la suite, il planifie et met en œuvre des approches afin de pousser (disséminer) les connaissances vers ce public, encore appelé utilisateurs de connaissances ${ }^{2}$. Le courtier de connaissances est un intermédiaire qui facilite la collaboration entre les producteurs et les utilisateurs des connaissances, il trouve des données scientifiques qui serviront à la prise de décisions, interprète et adapte les résultats de recherche en fonction du contexte local et cerne les nouveaux problèmes que la recherche peut contribuer à résoudre ${ }^{17}$. Son rôle est de créer des interactions entre les producteurs et les utilisateurs de connaissances. L'intervention pharmaceutique amène le pharmacien à collaborer avec les médecins (18\%), les infirmières (12\%) ou les deux (11\%). Les patients représentent les principaux utilisateurs des connaissances (74\%), suivis des professionnels de la santé (26\%). L'utilisateur des connaissances est une personne capable d'utiliser les connaissances issues de la recherche pour prendre des décisions éclairées au sujet de politiques, de programmes ou de pratiques en matière de santé. Le niveau de participation de l'utilisateur des connaissances au processus de recherche peut varier en intensité et en complexité, selon la nature de la recherche et les besoins de l'utilisateur de l'information ${ }^{18}$. Le pharmacien est également considéré comme un des utilisateurs des connaissances dans $19 \%$ des études.

Les interventions pharmaceutiques ciblent surtout des patients atteints de certaines pathologies (p. ex. diabète [19\%] ou encore hypertension [15\%]) tandis que $20 \%$ des interventions ne ciblent aucune affection en particulier. 


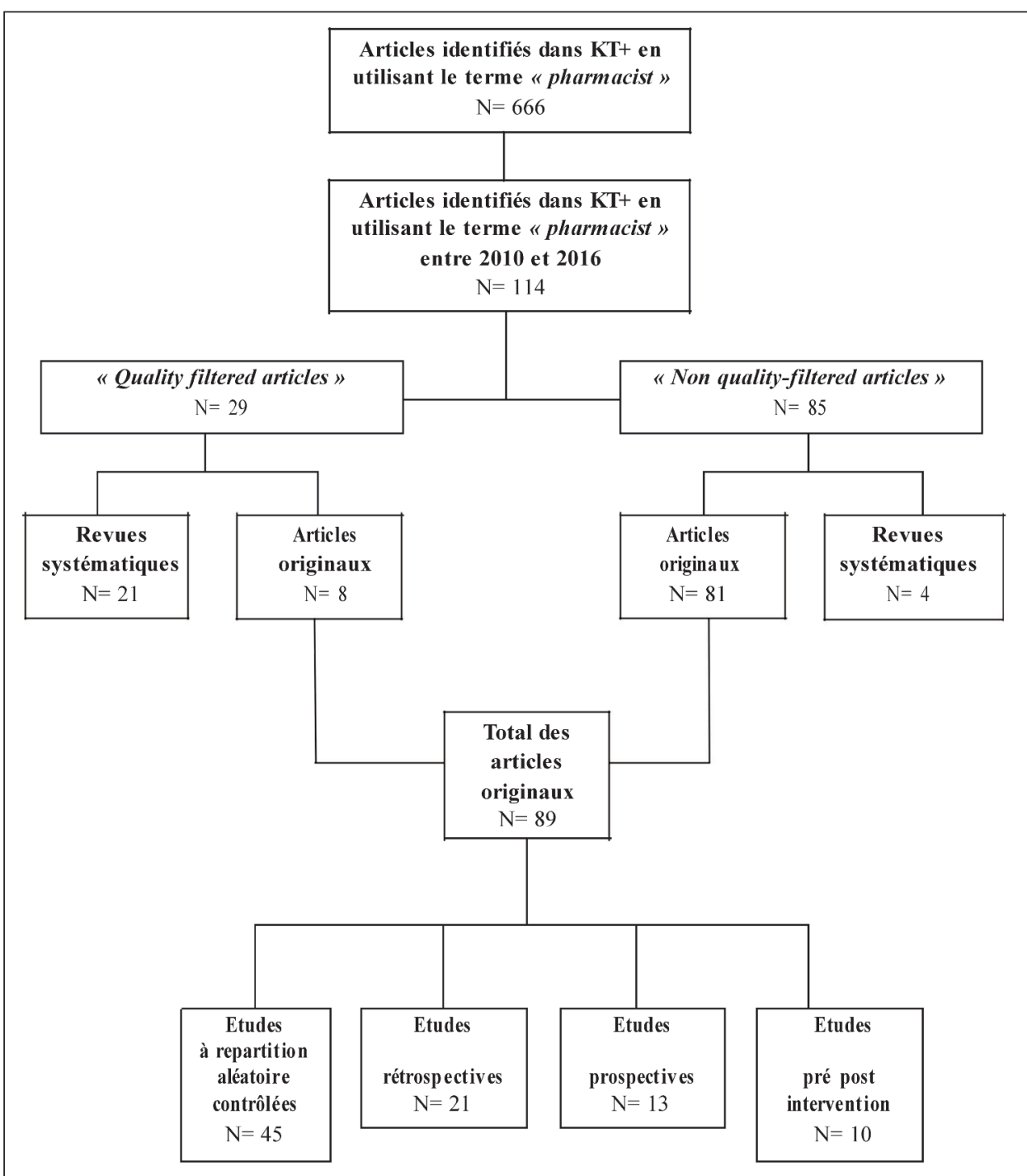

Figure 1. Diagramme de flux des articles sélectionnés.

La majorité des interventions concerne des étapes de soins pharmaceutiques et des séances d'éducation thérapeutique ou de conseils aux patients (64\%). L'éducation thérapeutique participe à l'amélioration de la santé du patient, de sa qualité de vie et de celle de ses proches ${ }^{19}$. Dans un premier temps, le professionnel de la santé réalise un "diagnostic éducatif " visant à déterminer les besoins et les attentes du patient, à formuler avec lui les compétences à acquérir et les priorités d'apprentissage. Dans un second temps, des séances d'éducation thérapeutique individuelles ou collectives sont mises en place pour permettre au patient d'atteindre ses objectifs. On parle également de gestion de la thérapie médicamenteuse (GTM ou medication therapy manage$m e n t)^{20-22}$. Celle-ci comporte la revue et l'ajustement de la thérapie médicamenteuse pour l'ensemble des médicaments lors de services (c.-à-d. dispensation de médicaments) ou de soins (c.-à-d. au chevet des patients) en fonction des besoins des patients, des cibles fixées par les médecins et autres cliniciens, de l'évolution clinique et des résultats observés sur la santé. Dans le cadre de cette démarche, le pharmacien prévient ou résout les problèmes potentiels et réels liés à la pharmacothérapie. Parmi les études originales, on trouve des interventions de GTM pour le diabète, l'hypertension, le VIH, la prise en charge de pathologies chroniques diverses lorsque les patients présentent plusieurs comorbidités, comme une dyslipidémie, le diabète, l'hypertension, les troubles de la coagulation, l'ostéoporose, la dépression, le delirium, l'insuffisance rénale chronique et l'utilisation d'érythropoḯtine ou encore en pédiatrie. Le pharmacien assure le suivi et l'interprétation des résultats biologiques, comme la mesure de la pression artérielle, le rapport international normalisé ou la glycémie. Cette surveillance se fait parfois à distance, comme le télémonitorage. La GTM est réalisée en collaboration avec les autres professionnels de la santé (p. ex. médecins, infirmières). 
Tableau 1 (partie 1 de 2). Profil des interventions pharmaceutiques mettant en valeur l'application des connaissances

\begin{tabular}{|c|c|c|c|c|c|}
\hline \multirow[b]{2}{*}{ Devis d'étude } & \multicolumn{4}{|c|}{ Type d'étude; nombre d'études } & \multirow[b]{2}{*}{$\begin{array}{c}\text { Nombre } \\
\text { total (\%) } \\
(n=89)\end{array}$} \\
\hline & $\begin{array}{l}\text { Étude contrôlée } \\
\text { à répartition } \\
\text { aléatoire } \\
(n=45)\end{array}$ & $\begin{array}{l}\text { Étude } \\
\text { prospective } \\
(n=13)\end{array}$ & $\begin{array}{l}\text { Étude } \\
\text { rétrospective } \\
(n=21)\end{array}$ & $\begin{array}{c}\text { Étude pré- } \\
\text { post-intervention } \\
(n=10)\end{array}$ & \\
\hline \multicolumn{6}{|l|}{ Lieux de l'intervention } \\
\hline Établissement de soins & 31 & 12 & 16 & 7 & $66(74)$ \\
\hline Réseau de soins communautaire & 14 & 1 & 5 & 3 & $23(26)$ \\
\hline \multicolumn{6}{|l|}{$\begin{array}{l}\text { Producteurs ou courtiers } \\
\text { de connaissances }\end{array}$} \\
\hline Pharmacien & 43 & 12 & 21 & 8 & $84(94)$ \\
\hline Médecin & 12 & 4 & 1 & 3 & $20(22)$ \\
\hline Médecin + pharmacien & 10 & 3 & 1 & 2 & $16(18)$ \\
\hline Infirmière & 8 & 3 & 0 & 3 & $14(16)$ \\
\hline Pharmacien + infirmière & 8 & 0 & 0 & 3 & $11(12)$ \\
\hline Médecin + pharmacien + infirmière & 6 & 2 & 0 & 2 & $10(11)$ \\
\hline \multicolumn{6}{|l|}{ Utilisateurs des connaissances } \\
\hline Patient & 40 & 6 & 17 & 3 & $66(74)$ \\
\hline Pharmacien & 7 & 3 & 3 & 4 & $17(19)$ \\
\hline Médecin & 3 & 4 & 1 & 8 & $16(18)$ \\
\hline Infirmière & 5 & 0 & 0 & 4 & $9(10)$ \\
\hline \multicolumn{6}{|l|}{$\begin{array}{l}\text { Pathologies et secteurs concernés } \\
\text { par l'intervention }\end{array}$} \\
\hline Diabète & 8 & 0 & 8 & 1 & $17(19)$ \\
\hline Hypertension & 9 & 2 & 2 & 0 & $13(15)$ \\
\hline Gériatrie & 6 & 2 & 0 & 1 & $9(10)$ \\
\hline Pédiatrie & 1 & 3 & 0 & 4 & $8(9)$ \\
\hline Maladies cardiovasculaires & 6 & 0 & 2 & 0 & $8(9)$ \\
\hline Anticoagulation & 3 & 0 & 2 & 0 & $5(6)$ \\
\hline Antibiothérapie et immunisation & 1 & 0 & 0 & 2 & $3(3)$ \\
\hline Insuffisance rénale chronique & 1 & 1 & 1 & 0 & $3(3)$ \\
\hline VIH & 0 & 0 & 2 & 0 & $2(2)$ \\
\hline Dépression & 1 & 0 & 0 & 0 & $1(1)$ \\
\hline Delirium & 1 & 0 & 0 & 0 & $1(1)$ \\
\hline Ostéoporose & 1 & 0 & 0 & 0 & 1 (1) \\
\hline $\begin{array}{l}\text { Aucune affection ciblée } \\
\text { avec précision }\end{array}$ & 7 & 5 & 4 & 2 & $18(20)$ \\
\hline \multicolumn{6}{|l|}{ Catégories d'interventions } \\
\hline Travailler en interdisciplinarité & 45 & 13 & 17 & 10 & $85(96)$ \\
\hline $\begin{array}{l}\text { Transfert de connaissances vers } \\
\text { le patient et les autres intervenants }\end{array}$ & 45 & 13 & 17 & 10 & $85(96)$ \\
\hline $\begin{array}{l}\text { Établir une relation de confiance } \\
\text { avec le patient et les autres } \\
\text { intervenants }\end{array}$ & 34 & 13 & 11 & 10 & $68(76)$ \\
\hline $\begin{array}{l}\text { Évaluer les besoins du patient et } \\
\text { de l'équipe traitante }\end{array}$ & 34 & 11 & 16 & 7 & $68(76)$ \\
\hline $\begin{array}{l}\text { Évaluer la pharmacothérapie et les } \\
\text { mesures non pharmacologiques }\end{array}$ & 34 & 6 & 19 & 3 & $62(70)$ \\
\hline Assurer le suivi des patients & 34 & 5 & 17 & 2 & $58(65)$ \\
\hline Demeurer compétent & 4 & 6 & 0 & 6 & $16(18)$ \\
\hline $\begin{array}{l}\text { Effectuer un bilan comparatif des } \\
\text { ordonnances }\end{array}$ & 2 & 3 & 1 & 1 & 7 (8) \\
\hline Gérer et préparer les médicaments & 3 & 1 & 0 & 0 & $4 \quad(4)$ \\
\hline
\end{tabular}


This single copy is for your personal, non-commercial use only.

For permission to reprint multiple copies or to order presentation-ready copies for distribution, contact CJHP at publications@cshp.ca

Tableau 1 (partie 2 de 2). Profil des interventions pharmaceutiques mettant en valeur l'application des connaissances

Type d'étude; nombre d'études

\begin{tabular}{|c|c|c|c|c|c|}
\hline Devis d'étude & $\begin{array}{l}\text { Étude contrôlée } \\
\text { à répartition } \\
\text { aléatoire } \\
(n=45)\end{array}$ & $\begin{array}{l}\text { Étude } \\
\text { prospective } \\
(n=13)\end{array}$ & $\begin{array}{c}\text { Étude } \\
\text { rétrospective } \\
(n=21)\end{array}$ & $\begin{array}{c}\text { Étude pré- } \\
\text { post-intervention } \\
(n=10)\end{array}$ & $\begin{array}{c}\text { Nombre } \\
\text { total (\%) } \\
(n=89)\end{array}$ \\
\hline \multicolumn{6}{|c|}{ Évaluation des objectifs primaires } \\
\hline $\begin{array}{l}\text { État clinique du patient ou } \\
\text { observance }\end{array}$ & 33 & 3 & 16 & 1 & $53(60)$ \\
\hline Prescription médicamenteuse & 10 & 6 & 5 & 5 & $26(29)$ \\
\hline Ressources (hospitalisation, coûts) & 15 & 4 & 5 & 1 & $25(28)$ \\
\hline $\begin{array}{l}\text { Niveau de connaissances des } \\
\text { professionnels santé }\end{array}$ & 1 & 3 & 0 & 4 & $8(9)$ \\
\hline Conformité à des lignes directrices & 3 & 0 & 0 & 0 & $3(3)$ \\
\hline \multicolumn{6}{|l|}{ Effet des interventions } \\
\hline Positif & 29 & 9 & 18 & 10 & $66(74)$ \\
\hline Neutre & 10 & 2 & 2 & 0 & $14(16)$ \\
\hline Négatif & 6 & 2 & 1 & 0 & $9(10)$ \\
\hline
\end{tabular}

Onze pour cent des études ciblent plus particulièrement cette collaboration pluridisciplinaire et l'optimisation de la collaboration entre professionnels de santé.

Les interventions du pharmacien portent sur la réduction des erreurs de prescription ou les effets indésirables par la réalisation de bilans comparatifs des médicaments (13\%), l'utilisation de logiciels de prescription, la vérification de l'ordonnance de sortie par le pharmacien, l'utilisation des critères STOPP/START en gériatrie, la formation des prescripteurs ou encore l'affectation d'un pharmacien dans le service pour vérifier les prescriptions.

D'autres interventions pharmaceutiques consistent à former les professionnels de la santé (11\%) sur l'utilisation des antibiotiques, l'administration de médicaments, la culture de la sécurité à l'hôpital, les pratiques d'analgésie, la vérification des signes vitaux du patient. De plus, les interventions pharmaceutiques visent à estimer l'adhésion aux lignes directrices sur l'asthme, l'antibiothérapie, la vaccination contre la grippe des professionnels de santé et l'effet d'incitations financières sur l'adhésion aux lignes directrices.

En utilisant les catégories d'interventions proposées par la plate-forme Impact Pharmacie, les interventions recensées impliquent le plus souvent plus d'une catégorie, de sorte que la somme des catégories dépasse largement $100 \%$. Toutes les interventions rapportées dans les études sont multiples, c.-à-d. qu'elles font appel à plusieurs types d'interventions.

Dans les études recensées, l'objectif primaire évalué porte sur l'état clinique des patients ou l'observance (60\%), la prescription médicamenteuse (29\%), les ressources (28\%), le niveau de connaissances des professionnels de la santé (9\%) ou encore l'adhésion à des lignes directrices (3\%). Les types de ressources évaluées concernent principalement la durée de l'hospitalisation et le coût des médicaments et des ressources professionnelles. L'impact des interventions pharmaceutiques se révèle positif $(74 \%)$, neutre $(16 \%)$ ou négatif $(10 \%)$. Le résultat qualifié de neutre signifie que l'intervention du pharmacien n'a généré aucun changement.

\section{Revues systématiques}

Des 114 études retenues, 25 sont des revues systématiques dont cinq méta-analyses. Elles proviennent des États-Unis ( $n=7,28 \%)$, du Royaume Uni $(n=4,16 \%)$, d'Irlande ( $n=2,8 \%)$, de Norvège $(n=1,4 \%)$, de Thaillande $(n=1,4 \%)$, de France $(n=1,4 \%)$, d'Espagne $(n=1,4 \%)$ et du Canada ( $n=1,4 \%)$. Sept études proviennent du groupe Cochrane réunissant des chercheurs de différents pays (28\%). Ces revues systématiques comportent un nombre variable d'études originales (c.-à-d. jusqu'à 20 études [36\%], entre 21 et 50 études [28\%], plus de 50 études [36\%]) et décrivent des interventions qui se déroulent surtout en établissement de santé (80\%).

Dans ces revues systématiques, le pharmacien est producteur ou courtier de connaissances dans $100 \%$ des études. L'intervention pharmaceutique amène le pharmacien à collaborer notamment avec les médecins (28\%) et les infirmières (28\%). Les patients représentent les principaux utilisateurs des connaissances $(72 \%)$, suivis des médecins ( $40 \%$ ) et des infirmières (40\%). Le pharmacien est également considéré comme un des utilisateurs des connaissances dans $28 \%$ des études.

Les interventions pharmaceutiques ciblent des populations et des affections particulières (p. ex. gériatrie [28\%], pathologies chroniques [12\%], anticoagulation [8\%], pédiatrie [8\%], insuffisance rénale chronique [8\%], hypertension [8\%], risque cardiovasculaire [4\%]) tandis que $24 \%$ des interventions ne ciblent aucune population ou affection particulière.

Les revues systématiques visent à évaluer le rôle du pharmacien dans l'amélioration de l'état clinique du patient à travers la GTM, la diminution des erreurs de prescription ou la survenue d'effets indésirables ou encore la formation des professionnels de la santé, par exemple pour les inciter à se faire vacciner contre la grippe.

Dans les revues systématiques, l'objectif primaire évalué porte principalement sur l'état clinique du patient ou l'adhésion (38\%) 
et la prescription médicamenteuse (38\%). L’impact des interventions pharmaceutiques se révèle positif ( $44 \%$ ) ou neutre (56\%).

\section{DISCUSSION}

\section{Que retenir de cette revue de la littérature scientifique?}

À partir de la plateforme $\mathrm{KT}+$, nous avons été en mesure de recenser 114 études décrivant des interventions pharmaceutiques publiées en sept ans de 2010 à 2016. Il s'agit d'une revue de littérature originale utilisant l'AC comme filtre spécifique de l'évaluation des répercussions de l'intervention des pharmaciens. Ce nombre d'études est relativement important étant donné qu'il représente $17 \%$ des articles de la plateforme $\mathrm{KT}+$ recensés lorsqu'on effectue une recherche avec le terme "pharmacist ". Compte tenu de la méthode proposée par cette équipe de recherche, il s'agit d'études comportant une méthodologie valable et des protocoles de recherche permettant d'évaluer les retombées de l'intervention pharmaceutique. À titre comparatif, la plate-forme Impact Pharmacie recense 892 études pour la même période d'étude. Ceci tient au fait que les critères d'inclusion de cette plateforme sont plus larges que ceux de $\mathrm{KT}+$ (p. ex. articles descriptifs, articles avec des retombées avec ou sans groupe contrôle).

La revue de la littérature révèle qu'une majorité des études originales prises en compte ont été réalisées en établissement de santé, où le pharmacien est à la fois producteur, courtier et utilisateur des connaissances. Il est souvent plus facile de mettre en pratique le concept d'AC en établissement de santé qu'en milieu communautaire, compte tenu de la présence d'équipes interdisciplinaires, d'activités d'enseignement et de recherche. Une partie du temps est consacrée aux échanges entre professionnels tandis que le pharmacien communautaire exerce souvent seul, aidé du personnel technique, et ses contacts avec les autres professionnels sont épisodiques et téléphoniques. Ainsi, il est plus difficile de réaliser des activités similaires en milieu communautaire où tous les intervenants exercent dans des lieux différents.

Les interventions pharmaceutiques en AC portent sur des pathologies chroniques (p. ex. diabète, hypertension, maladies cardiovasculaires, insuffisance rénale, ostéoporose, VIH, dépression) ou ciblent des patients exposées à la polypharmacie, comme la gériatrie.

Il existe deux principaux types d'interventions pharmaceutiques visant l'AC, le premier est orienté vers le patient et le second, vers les autres professionnels de santé. Ainsi, la majorité des interventions pharmaceutiques étudiées concernent les étapes dans la démarche de soins pharmaceutiques (c.-̀̀-d. établir une relation de confiance [76\%], effectuer un bilan comparatif des ordonnances [8\%], évaluer les besoins du patient [76\%], évaluer la pharmacothérapie et les mesures non pharmacologiques [70 \%], gérer et préparer les médicaments [5\%], assurer le suivi des patients [65\%]). La minorité des interventions portent sur le maintien de la compétence (18\%). Pour le second type, la majorité des interventions pharmaceutiques ciblent le travail en interdisciplinarité et le transfert des connaissances aux autres intervenants. Ce transfert contribue notamment à limiter les erreurs médicamenteuses et à optimiser le circuit du médicament.

La revue de la littérature montre que les études retenues comportent des objectifs primaires permettant d'évaluer des interventions pharmaceutiques dont l'impact est soit positif dans $74 \%$ des cas, soit neutre dans $16 \%$ des cas, soit négatif dans $10 \%$ des cas. Ces proportions sont relativement similaires à celles observées dans la plate-forme Impact Pharmacie depuis 1990 $(60 \%, 29 \%, 1 \%)^{23}$ ou à celles de l'étude de Tanguay et collab. ${ }^{24}$. Ainsi, dans environ deux-tiers des études, les auteurs sont en mesure de démontrer des répercussions favorables de l'intervention de pharmaciens.

\section{Que doit-on faire en recherche sur les pratiques pharmaceutiques?}

La recherche en pratique pharmaceutique doit encore relever de nombreux défis et cette revue documentaire met en évidence certains enjeux méthodologiques (p. ex. répartition aléatoire dans un contexte de soins, maintien de l'insu). En 2009, Charrois et collab. ont revu les enjeux en recherche appliquée $e^{25}$. Parmi les difficultés notées dans les revues systématiques publiées, la pauvreté de la description de l'intervention est évidente (intervenant, formation de base, rôle, nature de l'intervention, outils utilisés, etc.). Il existe différents outils de planification d'une intervention, surtout si elle fait l'objet d'une évaluation structurée et d'une publication ultérieure (p. ex. Template for Intervention Description and Replication (TIDieR) Checklist and Guide) ${ }^{26}$. Un autre groupe de recherche brésilien a proposé un outil appliqué aux interventions pharmaceutiques (Descriptive Elements of Pharmacist Intervention Characterization Tool-DEPICT) ${ }^{27}$.

Grimshaw et collab. soulignent que l'absence de standardisation dans les études portant sur l'AC peut compliquer le choix du type d'intervention à développer à cause du manque de données systématiques portant sur les interventions, l'absence de cadre de planification rigoureux, la réalisation et la communication des résultats des interventions ${ }^{28}$. De plus, souvent les résultats des études ne peuvent pas être utilisés dans leur intégralité, car les interventions sont peu ou mal décrites, s'appuient rarement sur une base théorique, et il n'existe pas de guide pratique permettant de les appliquer dans un nouveau contexte.

Ainsi, la recherche en $\mathrm{AC}$ se heurte entre autres à la difficulté de mettre en place une standardisation de la description des interventions dans le but de faciliter l'établissement des déterminants (obstacles et facteurs favorisant le succès de l'intervention) ainsi que la comparaison de ceux-ci entre différentes études, de comprendre comment l'intervention est construite et sur quel mécanisme elle repose pour pouvoir ensuite la reproduire dans un autre contexte.

De même, le rapport de l'Institut national de santé mentale sur les progrès en science de l'AC paru en 2004 appelle à des avancées dans la définition, la description et l'évaluation des 
interventions ${ }^{29}$. Le groupe WIDER (Workgroup for Intervention Development and Evaluation Research) a également fait paraitre des recommandations destinées aux chercheurs voulant publier dans des journaux scientifiques afin qu'ils optimisent la description des interventions liées aux changements de comportement ${ }^{30}$. Walter et collab. proposent une taxonomie des interventions ${ }^{31}$. Enfin, Proctor et collab. ont proposé des recommandations pour harmoniser les descriptions des stratégies de mise en œuvre (composantes, méthodes de réalisation), pour faciliter leur mise en place et leur évaluation en $\mathrm{AC}^{32}$. Ils recommandent de nommer l'intervention, de lui attribuer une définition et de la décrire en détail. Cette dernière étape repose sur sept dimensions, soit l'acteur (celui qui délivre l'intervention), l'action (description de l'intervention), les cibles de l'action (public visé, connaissances transférées), la temporalité (moment de l'intervention elle-même), la dose (fréquence de l'intervention), l'objectif primaire (impact), la justification (théorie, modèle, preuve empirique, cadre conceptuel sur lequel repose l'intervention).

\section{Que faire en pratique au sein du département de pharmacie?}

Notre revue documentaire et notre réflexion mettent en évidence les éléments suivants : il faut encourager l'intégration des concepts de l'AC et des théories du changement comportemental dans le cursus de pharmacie, désigner un pharmacien responsable de l'AC au sein de chaque département de pharmacie, encourager ce pharmacien à participer à des communautés de pratique et intégrer l'AC dans le plan de communication du département et dans les activités d'évaluation des pratiques pharmaceutiques. Nous pensons qu'un modèle en quatre étapes d'AC (c.-à-d. analyse du contexte, synthèse des connaissances, partage des connaissances, évaluation de l'intervention) répond aux besoins de la pratique pharmaceutique. Les IRSC proposent une liste de vérification en neuf étapes pour guider les professionnels de la santé désireux de mettre en place des interventions en $\mathrm{AC}^{33}$. L'utilisation de cet outil au sein du département de pharmacie pourrait améliorer la formation des pharmaciens, résidents et étudiants en pharmacie ainsi que des assistants techniques. Par ailleurs, il faudrait créer des projets transversaux visant à optimiser la qualité des soins prodigués aux patients et l'accès à l'innovation entre le département de pharmacie et les services de l'hôpital. Cet outil pourrait aussi servir de base à l'élaboration d'un plan de communication du département de pharmacie. Ce plan devrait englober des modes de communication efficaces et multiples pour rejoindre les différents publics et des objectifs spécifiques (p. ex. augmentation de la conformité de $X \%$ pour l'utilisation d'un médicament $Y$ dans telle situation). De plus, la réalisation d'interventions d'AC impose la mise en place d'une gestion de l'information. Une bonne gestion des informations requiert une sélection des sources d'informations selon des critères objectifs (c.-à-d. rigueur et justesse du contenu scientifique, actualisation des données, auteurs) puis une régulation de la consultation de ces informations (c.-à-d. allocation d'un temps prédéterminé) et une prise de décision sur la possibilité et l'intérêt de la mise en pratique des informations retenues.

Cette revue documentaire comporte des limites. Notre stratégie de recherche ne cible que la plateforme KT+. Une recherche dans Pubmed, Embase, Google Scholar et parmi les revues non indexées permettrait sans doute de recenser un nombre plus élevé d'études originales. Toutefois, comme il existe des dizaines de termes relatifs à l'AC, l'adoption d'une définition claire et opérationnelle serait nécessaire pour compléter la sélection et la prise en compte des études. À titre de revue documentaire exploratoire sur la thématique de l'AC, cette revue de la littérature constitue un point de départ intéressant pour encourager davantage la recherche sur le sujet.

\section{CONCLUSION}

Cette revue de la littérature scientifique décrit le profil des interventions visant l'AC en pratique pharmaceutique. Le pharmacien est le principal producteur de connaissances et oriente ses interventions vers les patients ou les professionnels de santé.

Le transfert des connaissances contribue notamment à limiter les erreurs médicamenteuses et à optimiser le circuit du médicament. La majorité des interventions concerne la démarche de soins pharmaceutiques et cible le travail en interdisciplinarité L'impact de ces interventions est positif dans la majorité des études. Cependant, le manque de rigueur méthodologique lors de leur conception et la disparité de leur description limite l'exploitation des résultats et la reproductibilité des interventions dans d'autres contextes. La standardisation de la description des interventions pourrait permettre de faire progresser la recherche sur la science de l'AC.

Les pharmaciens devraient prendre conscience qu'ils sont les acteurs de l'AC dans leur activité professionnelle quotidienne afin de devenir proactifs en la matière. À l'avenir, trois mesures pourraient contribuer à soutenir le développement de l'AC en pharmacie : la mise en place d'une formation initiale et continue, la gestion de l'information et la désignation d'un pharmacien responsable de l'AC au sein de chaque département de pharmacie. Une formation continue constituée d'activités d'autoapprentissage et de conférences offertes par nos associations provinciales ou nationales pourrait permettre de rejoindre un large public. Ces initiatives devraient servir à encourager la recherche et à promouvoir la mise en place de stratégies par les pharmaciens. Le concept d'AC peut être utile pour soutenir la création d'un modèle de pratique pharmaceutique cohérent.

\section{Références}

1. L'application de connaissances : définition. Ottawa $(\mathrm{ON})$ : Instituts de recherche en santé du Canada; 28 juillet 2016. Publié au : www.cihrirsc.gc.ca/f/29418.html. Consulté le 20 juin 2017.

2. La dissémination et l'échange des connaissances. Ottawa $(\mathrm{ON})$ : Instituts de recherche en santé du Canada; 9 août 2010. Publié au : www.cihrirsc.gc.ca/f/41953.html. Consulté le 20 juin 2017.

3. Curran GM, Bauer M, Mittman B, Pyne JM, Stetler C. Effectivenessimplementation hybrid designs: combining elements of clinical effectiveness and implementation research to enhance public health impact. Med Care. 2012;50(3):217-26. 
4. KT science terms. Hamilton (ON): WhatisKT; 2017. Publié au : http://whatiskt.wikispaces.com/KT+Science+Terms. Consulté le 20 juin 2017.

5. Effective practice and organisation of care (EPOC). EPOC taxonomy topics list. Cochrane Collaboration; 2015. Publié au : http://epoc.cochrane. org/sites/epoc.cochrane.org/files/public/uploads/epoc_taxonomy_13.12.16. pdf. Consulté le 20 juin 2017.

6. Baker R, Camosso-Stefinovic J, Gillies C, Shaw EJ, Cheater F, Flottorp S, et al. Tailored interventions to overcome identified barriers to change: effects on professional practice and health care outcomes. Cochrane Database Syst Rev. 2010;(3):CD005470.pub2.

7. Grimshaw JM, Thomas RE, MacLennan G, Fraser C, Ramsay CR, Vale L, et al. Effectiveness and efficiency of guideline dissemination and implementation strategies. Health Technol Assess. 2004;8(6):iii-iv, 1-72.

8. Hakkennes S, Dodd K. Guideline implementation in allied health professions: a systematic review of the literature. Qual Saf Health Care. 2008;17(4):296-300.

9. LaRocca R, Yost J, Dobbins M, Ciliska D, Butt M. The effectiveness of knowledge translation strategies used in public health: a systematic review. BMC Public Health. 2012;12:751.

10. Prior M, Guerin M, Grimmer-Somers K. The effectiveness of clinical guideline implementation strategies - a synthesis of systematic review findings. J Eval Clin Pract. 2008;14(5):888-97.

11. Scott SD, Albrecht L, O'Leary K, Ball GDC, Hartling L, Hofmeyer A, et al. Systematic review of knowledge translation strategies in the allied health professions. Implement Sci. 2012;7(1):70.

12. KT+: knowledge translation. About this site. Hamilton $(\mathrm{ON}):$ McMaster University; 2017. Publié au : https://plus.mcmaster.ca/kt/Pages/About. Consulté le 20 juin 2017.

13. KT+: knowledge tranlation. Inclusion criteria. Hamilton $(\mathrm{ON}):$ McMaster University; $1^{\text {er }}$ février 2017. Publié au : http://hiru.mcmaster.ca/hiru/ InclusionCriteria.html. Consulté le 20 juin 2017.

14. KT+: knowledge translation. List of journals. Hamilton $(\mathrm{ON}):$ McMaster University; 2017. Publié au : http://hiru.mcmaster.ca/hiru/JournalsList.asp. Consulté le 20 juin 2017.

15. Impact pharmacie. Montréal (QC) : Unité de recherche en pratique pharmaceutique du CHU Sainte Justine; 2017. Publié au : www.impactpharmacie.org/. Consulté le 20 juin 2017.

16. Thomas M, Dhanani S, Irwin D, Writer H, Doherty D. Development, dissemination and implementation of a sedation and analgesic guideline in a pediatric intensive care unit ... it takes creativity and collaboration. Dynamics. 2010;21(4):16-25.

17. Lavis JN, Robertson D, Woodside JM, McLeod CB, Abelson J; Knowledge Transfer Study Group. How can research organizations more effectively transfer research knowledge to decision makers? Milbank Q. 2003;81(2): 221-48, 171-2.

18. Engagement des utilisateurs de connaissances. Ottawa $(\mathrm{ON})$ : Instituts de recherche en santé du Canada; 2017. Publié au : www.cihr-irsc.gc.ca/f/ 49505.html. Consulté le 20 juin 2017.

19. Éducation thérapeutique du patient : comment la proposer et la réaliser? Saint-Denis (France) : Haute autorité de santé; juin 2007. Publié au : https://www.has-sante.fr/portail/upload/docs/application/pdf/etp_-comment_la_proposer_et_la_realiser_-_recommandations_juin_2007.pdf. Consulté le 20 juin 2017

20. APhA MTM Central. Washington (DC) : American Pharmacists Association; 2017. Publié au : http://pharmacistsprovidecare.com/mtm. Consulté le 8 mai 2018 .

21. Ramalho de Oliveira D, Brummel AR, Miller DB. Medication Therapy Management: 10 years of experience in a large integrated health care system. J Manag Care Pharm. 2010;16(3):185-95.

22. Standards de pratique. Montréal (QC) : Ordre des pharmaciens du Québec; 2011. Publié au : https://www.opq.org/fr-CA/publications/standards-depratique/. Consulté le 20 juin 2017.

23. Barbier A, Girard S, Garnier J, Nugyen KD, Jaatoul M, Bergeron M, et al. Impact Pharmacie: une plateforme web sur les rôles et retombées du pharmacien- résultats de la phase III (IMPACTPHARMACIE36) [affiche]. Congrès Hopipharm; Nancy, France; 10-12 mai 2017.
24. Tanguay C, Guérin A, Bussières JF. Caractéristiques des revues systématiques présentant les interventions de pharmaciens. Ann Pharm Fr. 2014;72(6): 429-39.

25. Charrois TL, Durec T, Tsuyuki RT. Systematic reviews of pharmacy practice research: methodologic issues in searching, evaluating, interpreting, and disseminating results. Ann Pharmacother. 2009;43(1):118-22.

26. Hoffman JR, Kanzaria HK. Intolerance of error and culture of blame drive medical excess. BMJ. 2014;349(7979):g5702.

27. Rotta I, Salgado TM, Felix DC, Souza TT, Correr CJ, Fernandez-Llimos F. Ensuring consistent reporting of clinical pharmacy services to enhance reproducibility in practice: an improved version of DEPICT. $J$ Eval Clin Pract. 2015;21(4):584-90.

28. Grimshaw JM, Eccles MP, Lavis JN, Hill SJ, Squires JE. Knowledge translation of research findings. Implement Sci. 2012;7(1):50.

29. Advancing the science of implementation: improving the fit between mental health intervention development and service systems. Washington (DC) : National Institute of Mental Health; 2006. Publié au : www.nimh.nih.gov/ scientificmeetings/scienceofimplementation.pdf. Consulté le 20 juin 2017.

30. WIDER recommendations for reporting of behaviour change interventions. Equator Network; 2014. Publié au : https://www.equator-network.org/ reporting-guidelines/wider-recommendations-for-reporting-of-behaviourchange-interventions/. Consulté le 20 juin 2017.

31. Walter I, Nutley S, Davies H. Developing a taxonomy of interventions used to increase the impact of research. University of St. Andrews, Department of Management, Research Unit for Research Utilisation; février 2003. Publié au : https://www.st-andrews.ac.uk/ ruruweb/pdf/Taxonomy\%20 development\%20paper\%20070103.pdf. Consulté le 20 juin 2017.

32. Proctor EK, Powell BJ, McMillen JC. Implementation strategies: recommendations for specifying and reporting. Implement Sci. 2013;8(1):139.

33. Passer à l'action: nous connaissons les pratiques que nous souhaitons changer. Que faire, maintenant? Guide de mise en oeuvre pour les professionnels de la santé. Ottawa $(\mathrm{ON})$ : Instituts de recherche en santé du Canada; 4 septembre 2012. Publié au : www.cihr-irsc.gc.ca/f/45669.html. Consulté le 20 juin 2017.

Apolline Adé est candidate au D. Pharm., Faculté de pharmacie, Université Paris Descartes, Paris, France, et assistante de recherche, Unité de recherche en pratique pharmaceutique, Centre hospitalier universitaire Sainte-Justine, Montréal, Québec.

Denis Lebel, B. Pharm., M.Sc., FCSHP, est Adjoint au chef, Département de pharmacie et Unité de recherche en pratique pharmaceutique, Centre hospitalier universitaire Sainte-Justine, et Professeur titulaire de clinique, Faculté de pharmacie, Université de Montréal, Montréal, Québec.

Jean-François Bussières, B. Pharm., M. Sc., MBA, FCSHP, est Chef, Département de pharmacie et Unité de recherche en pratique pharmaceutique, Centre hospitalier universitaire Sainte-Justine, et Professeur titulaire de clinique, Faculté de pharmacie, Université de Montréal, Montréal, Québec.

Intérêts concurrents : Aucun déclaré.

Addresse de correspondance :

Jean-François Bussières

Département de pharmacie

Centre hospitalier universitaire Sainte-Justine

3175, chemin de la Côte Sainte-Catherine

Montréal QC H3T 1C5

Courriel : jf.bussieres@ssss.gouv.qc.ca

Financement : Aucun financement en relation avec le présent article n'a été déclaré par les auteurs. 